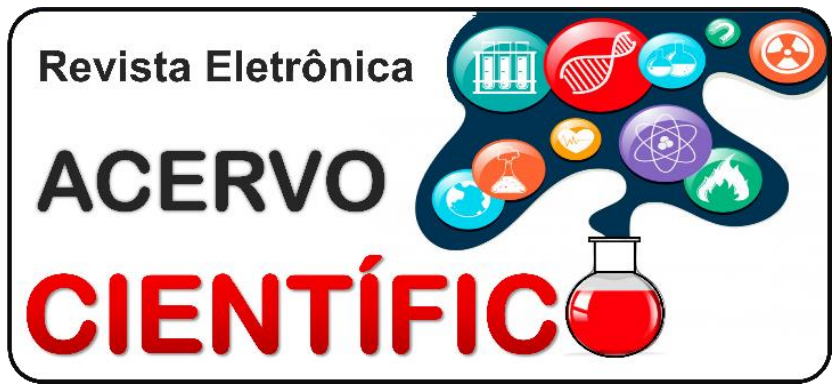

\section{REVISÃO BIBLIOGRÁFICA}

Recebido em: 10/2020

Aceito em: $11 / 2020$

Publicado em: 2/2021

\title{
Aleitamento materno: consequências do desmame precoce e o papel da enfermagem: uma revisão bibliográfica
}

\author{
Breastfeeding: consequences of early weaning and the role of nursing: a bibliographic \\ review
}
Lactancia materna: consecuencias del destete temprano y el papel de la enfermería: una revisión bibliográfica

Adriana dos Santos Oliveira ${ }^{1 *}$, Francieli Carniel ${ }^{1}$.

\begin{abstract}
Resumo: Este estudo objetivou reunir os resultados de pesquisas sobre os fatores que influenciam no desmame precoce. Trata-se de uma revisão integrativa da literatura cuja coleta e análise de dados foram realizadas nos meses de junho a agosto de 2020. Ao realizar o cruzamento dos descritores obteve-se um total de 3105 estudos, entre os anos de 2015 e 2020, estes passaram por um filtro e foram lidos manualmente, chegando ao total de 26 artigos. Os principais resultados desses artigos foram sintetizados em 3 (três) categorias temáticas para melhor compreensão do conteúdo proposto: Fatores relacionados ao recém-nascido, fatores relacionados aos pais e Fatores Externos. As variáveis envolvendo a criança foram: alterações fisiológicas no recém-nascido e a recusa do seio por parte da criança. Com relação aos pais, se destacou os fatores sociodemográficos, a idade materna e a crença do leite ineficiente. Já dentre as variáveis externas, o retorno ao trabalho ou estudo foi o predominante. Portanto, torna-se necessário o fortalecimento de ações comunitárias, reorientação dos serviços de saúde, orientações às nutrizes e na formação e articulação de redes de apoio a esta pratica a fim de promover resultados que possam contribuir à promoção de saúde da mãe-filho.
\end{abstract}

Palavras-chave: Desmame precoce, Aleitamento materno, Assistência de enfermagem.

\begin{abstract}
This study aimed to gather the results of research on the factors that influence early weaning. It is an integrative literature review whose data collection and analysis were carried out from June to August 2020. When crossing the descriptors, a total of 3105 studies were performed, between the years 2015 and 2020 , these they passed through a filter and were read manually, reaching a total of 26 articles. The main results of the articles were summarized in 3 (three) thematic categories for a better understanding of the proposed content: Factors related to birth, factors related to parents and External Factors. The variables involving the child were: physiological changes in the newborn and the child's refusal of the breast. Regarding parents, sociodemographic factors, maternal age and inefficient milk change were highlighted. Among the external variables, the return to work or study for the predominant. Therefore, it is necessary to strengthen community actions, reorient health services, guidance to nursing mothers and in the formation and articulation of support networks for this practice in order to promote the results that can be promoted to the health of the mother-child.
\end{abstract}

Keywords: Early weaning, Breast feeding, Nursing care.

${ }^{1}$ Centro Universitário São Lucas de Ji-Paraná. Ji-Paraná - RO. *E-mail: adrianaoliversantos@hotmail.com 
Resumen: Este estudio tuvo como objetivo recoger los resultados de la investigación sobre los factores que influyen en el destete precoz. Se trata de una revisión integradora de la literatura cuya recolección y análisis de datos se realizó de junio a agosto de 2020. Al cruzar los descriptores se realizaron un total de 3105 estudios, entre los años 2015 y 2020, estos pasaron por un filtro y se leyeron manualmente, alcanzando un total de 26 artículos. Los principales resultados de los artículos se resumieron en 3 (tres) categorías temáticas para una mejor comprensión del contenido propuesto: Factores relacionados con el nacimiento, factores relacionados con los padres y Factores Externos. Las variables que involucraron al niño fueron: cambios fisiológicos en el recién nacido y rechazo del pecho por parte del niño. En cuanto a los padres, se destacaron factores sociodemográficos, edad materna y cambio de leche ineficiente. Entre las variables externas, la reincorporación al trabajo o estudio para la predominante. Por ello, es necesario fortalecer las acciones comunitarias, reorientar los servicios de salud, la orientación a las madres lactantes y en la formación y articulación de redes de apoyo a esta práctica con el fin de promover los resultados que se puedan promover a la salud materno infantil.

Palabras clave: Destete temprano, Lactancia materna, Atención de enfermería.

\section{INTRODUÇÃO}

O Aleitamento Materno tem relevância no combate a fome extrema e desnutrição estabelecida nos dois primeiros anos de vida, sendo mesmo responsáveis pela sobrevivência da criança, principalmente aquelas em condições desfavoráveis. $E$ isso ocorre devido às inúmeras vantagens imunológicas e psicológicas (DIAS RB, et al., 2016; OLIVEIRA CS, et al., 2015).

A Organização Mundial de Saúde (OMS) e o Ministério da Saúde (MS) recomendam que os recémnascidos (RN) recebam o aleitamento materno exclusivo (AME) até os 6 meses de vida e o aleitamento materno (AM) até os 2 anos de idade (BRASIL, 2015; OMS, 2009).

No entanto, a prática de amamentação exclusiva até os seis meses no Brasil, ainda permanece longe de atingir o patamar ideal preconizado pelo United Nations International Children's Emergency Fund (UNICEF), da OMS e do MS. Segundo dados da II Pesquisa de Prevalência do AM nas capitais brasileiras e Distrito Federal, realizada em 2008, apontaram que apenas $41,0 \%$ das crianças até 6 meses de vida recebiam AME. Dentre as regiões, o Norte foi a que apresentou a maior prevalência $(45,9 \%)$ e os indicadores mais baixos foram apresentados na região nordeste (37\%). Ou seja, mesmo na região mais prevalente, não foi atingido a meta de $50 \%$ de AME preconizado pela OMS, o que torna a situação preocupante (MARGOTTI E e MATTIELLO R, 2016; PRADO CVC, et al., 2016).

Nestas condições, os profissionais de saúde que fazem parte dessas instituições são responsáveis por promover, apoiar e proteger a prática do aleitamento materno e apoiar as nutrizes para evitar o desmame precoce no Brasil. Nessa perspectiva, o(a) enfermeiro(a) da equipe de saúde tem um papel primordial frente à amamentação, pois são eles quem mais se relacionam com a mulher durante o ciclo gravídico-puerperal, principalmente no pré-natal, lidando com as demandas do aleitamento e são por meio de suas práticas que as nutrizes a aperfeiçoam a amamentação, melhorando assim, os índices de aleitamento materno, e consequentemente, diminuindo as taxas de desnutrição infantil, alergias, anemias, doenças dentárias e infecções que podem elevar à mortalidade infantil, além de diminuir as internações, custos com consultas, medicamentos e outros (MARGOTTI E e MATTIELLO R, 2016; MARINHO MS, et al., 2015).

Embora haja a existência de diversos estudos sobre o tema, ainda há o desafio de reunir e sintetizar as informações disponíveis para contribuir com sua aplicabilidade. Assim sendo, a pergunta norteadora da busca foi: $O$ que está posto na literatura como fatores que interferem o aleitamento materno, quais motivos levam ao desmame precoce e qual a função da enfermagem em tudo isso? A partir de tal questão levantada, este estudo tem por objetivo reunir os resultados de pesquisas sobre os fatores mais citados na literatura que influenciam no desmame precoce e o impacto do profissional de enfermagem nesse processo. 


\section{MÉTODOS}

Trata-se de um artigo de revisão integrativa da literatura, cuja elaboração da pesquisa utilizou-se das seguintes etapas: determinação do tema e definição da pesquisa, definição da questão da pesquisa, estabelecimento dos critérios de inclusão e exclusão (amostra), definição das categorias/informações a serem retiradas dos artigos utilizados como subsídios: autor, ano, título, revista, objetivo e desfecho.

A coleta de dados foi realizada nos meses de junho e julho de 2020 e a análise dos mesmos no mês de agosto de 2020, onde foram acessados os artigos publicados no período de 2015 a 2020, indexados nas bases de dados da Literatura Latino Americana e do Caribe em Ciências da Saúde (LILACS), Medical Literature Analysis and Retrieval System Online (MEDLINE) através da Biblioteca Virtual em Saúde (BVS), Scientific Electronic Library Online (SCIELO) e periódicos da Coordenação de Aperfeiçoamento de Pessoal de Nível Superior/Ministério da Educação (CAPES/MEC).

Para a busca dos artigos, foram utilizados descritores selecionados mediante consulta aos Descritores em Ciências da Saúde (DeCS) e com a combinação dos operadores boleanos "aleitamento materno exclusivo" AND "lactente" AND "desmame precoce" AND "breast feeding" and Infant AND "Early weaning". Os critérios de inclusão foram: pesquisas publicadas em português, inglês ou espanhol em formato de artigos entre os anos de 2015 e 2020. Como critérios de exclusão: optou-se pela não utilização de teses, dissertações ou monografias, bem como trabalhos que não apresentasse o artigo na íntegra nas bases de dados.

Ao realizar o cruzamento dos descritores obteve-se um total de 3105 estudos, depois de realizado o filtro obteve-se a amostra de 200 artigos, estes foram lidos título e resumos para identificar aqueles que se relacionavam com a temática do estudo, identificando ao final o total de 26 artigos, sendo cinco estudos da SCIELO, seis da LILACS, doze do CAPES e três da MEDLINE.

Prosseguindo com a organização dos estudos conciliados em tabelas, possibilitando uma visão mais ampla das principais temáticas abordadas, consequentemente possibilitou a extração das informações mais relevantes para esta revisão.

\section{RESULTADOS}

A amostra foi composta por 26 artigos, dos quais dezessete (17) estavam disponíveis na língua portuguesa, seis (6) foram escritos em espanhol e (3) em inglês. Quanto ao período de publicação, constatou-se que os anos que apresentaram maior número de artigos publicados foi 2015, com oito publicações, correspondendo a $30 \%$ das incluídas no estudo. Seguido pelo ano 2016 com cinco publicações, o que corresponde a 19\%. O ano de 2019 e 2018 possuem quatro estudos, o que representa $15 \%, 2017$ com três estudos (15\%) e por fim, o ano de 2020 com dois artigos (7\%).

Em relação ao delineamento de pesquisa, identificou-se que das 26 publicações, 12 (doze) utilizaram um método quantitativo (46\%), 10 (dez) eram estudos qualitativos (38\%) e 4 (cinco) eram revisões integrativas da literatura (15\%).

Os principais resultados desses artigos foram sintetizados em 3 (três) Categorias Temáticas para melhor compreensão do conteúdo proposto. Categoria I - fatores do recém-nascido, Categoria II -fatores relacionados aos pais, Categoria III —Fatores Externos, que estão apresentadas na Tabela 1. Visto que um artigo pode trazer uma ou várias características, a frequência com que as variáveis se repetiram nas diferentes pesquisas foi representado pelo número de artigos ( $n$ ) mostrado na coluna e pela sua porcentagem (\%). 
Tabela 1 - Síntese das categorias Temáticas após análise dos artigos da Revisão Integrativa.

\begin{tabular}{lcc}
\hline Categorias temáticas & Frequência №26 & $\%$ \\
\hline Fatores Relacionados à Criança & & \\
\hline Alteração fisiológica que afete o recém-nascido na & & 4 \\
hora de mamar (doença, hospitalização, baixo peso & & 8 \\
ao nascer). & 2 & 42 \\
Recusa ao seio por parte da criança. & 11 & 38 \\
\hline Fatores Relacionados aos Pais & 10 & 38 \\
\hline Mãe jovem e inexperiente (primigesta). & 10 & 38 \\
Baixa renda. & 10 & 30 \\
Baixa escolaridade. & 8 & 23 \\
Crença do leite fraco e ineficiente. & 6 & 15 \\
Trauma Mamilar ou dor ao Amamentar. & 4 & 15 \\
Uso de Bicos artificiais. & 4 & 42 \\
Depressão Materna. & & 19 \\
Parto cesáreo. & 11 & 23 \\
\hline Escolaridade & 5 & 7 \\
\hline Retorno ao trabalho ou Estudo. & 6 & \\
Interferência Familiar & 2 & \\
Profissionais de saúde ineficientes. & & \\
Pré-natal tardio; Pré-Natal realizado em instituição & & \\
privada. & & \\
\hline
\end{tabular}

Dentre os fatores relacionados à criança, 3\% estava relacionado às alterações fisiológicas, tais como: boca demasiado pequena, bebês imaturos que ainda não desenvolveram o reflexo de sucção; ou algumas iatrogenias decorridas do parto, como luxação de ombros, que produz desconforto no bebê dependendo da posição e, consequentemente, faz com que não recuse o seio, relatado em $7 \%$ dos artigos selecionados.

Já entre os fatores relacionados aos pais, o mais relatado foi a idade materna, aproximadamente $42 \%$, seguido pelas variáveis: baixa renda, baixa escolaridade e crença do leite fraco e ineficiente, com $38 \%$ cada. O trauma mamilar ou dor ao amamentar foi descrito em 8 dos 26 artigos selecionados, o uso de bicos artificiais em 6 dos 26, e a depressão materna e o parto cesáreo, ambos foram descritos em apenas $15 \%$ das pesquisas selecionadas para o presente estudo. Entre as variáveis externas ou ambientais, o retorno ao trabalho ou ao estudo foi o fator predominante, sendo citado em 11 dos 26 artigos. Os demais citados foram: Interferência familiar (19\%), profissionais de saúde ineficientes (23\%) e o pré-natal tardio e/ou realizado em instituição privada $(7 \%)$.

\section{DISCUSSÃO}

O aleitamento materno exclusivo (AME) que consiste na oferta de apenas leite materno aos lactentes, sem ingestão de alimentos sólidos ou outros líquidos como água e suco, sendo apenas permitida a ingestão de medicamentos e/ou complexo vitamínico é o recomendado pela Organização Mundial de Saúde e pelo Ministério da Saúde até os seis meses de idade da criança (LIMA APC, et al., 2018; MONTEIRO JRS, et al., 2020). Mas são diversos os fatores que podem ocasionar o desmame precoce da criança ou a suplementação com outros alimentos. Dentre eles, variáveis envolvendo a própria criança, os pais e fatores ambientais ou externos.

Os fatores para o desmame precoce envolvendo a criança, estão relacionadas às alterações fisiológicas que interferem tanto na amamentação do lactente, quanto em seu desejo por amamentar. Entre as características relacionadas ao recém-nascido $(R N)$ estão as variáveis: Alterações fisiológicas que afetem o RN na hora de mamar, tais como doenças, hospitalização e baixo peso ao nascer; e a recusa ao seio por parte da criança (ALVARENGA SC, et al., 2017; MONTESCHIO CAC, et al., 2015).

$O$ desinteresse ou a recusa do bebê quanto à amamentação pode contribuir na interrupção da amamentação exclusiva de forma precoce. Tal rejeição pode estar relacionada a uma má postura ou a causas morfológicas, tais como: a criança possuir uma boca pequena ou a mama da mãe ser grande com 
uma aréola tensa o que ocasiona em um peito mais plano, dificultando na pega correta; além do atraso no desenvolvimento do reflexo de sucção em alguns RNs; ou algumas latrogenias resultantes do parto, como luxação de ombros, que a depender da posição produz desconforto no bebê e, consequentemente, faz com que não possua a vontade de mamar (ALVARENGA SC., 2017).

É válido ressaltar que nos artigos selecionados para o presente estudo, os fatores relacionados à criança foram menos discutidos que os demais. Isso pode ser explicado pelo fato de a maior parte dos artigos darem maior enfoque às variáveis tanto internas quanto externas que acometem as nutrizes. Enquanto que nas crianças, o assunto mais abordado foi relacionado aos benefícios da amamentação e as consequências do desmame precoce.

Dentre os fatores de risco relacionados aos pais, a variável que mais se acentua é o sociodemográfico (renda encontrado em $38 \%$ dos artigos e escolaridade em $38 \%$ dos artigos pesquisados) que foi citado em $76 \%$ dos artigos selecionados. As mulheres que possuem um nível de instrução mais alto associado a uma maior renda, tendem a expressar mais leite materno, bem como manter a AME até os seis meses com mais frequência que as aquelas com um menor grau de escolaridade. Uma das explicações possíveis é que mulheres com mais tempo de estudo tendem a procurar mais informações acerca da amamentação. Além disso, por possuírem maior renda, tem mais acesso a recursos tecnológicos e humanos para as auxiliarem no período de amamentação (CRISTOFARI RC, et al., 2019; GONÇALVES GP, et al., 2019; HAS EMM, et al., 2018; PANG WW, et al., 2017).

Outro fator de risco muito citado na literatura é a idade materna associada à primigestação (GONÇALVES GP, et al., 2019). Há uma maior tendência de interrupção do AME em mulheres mais jovens. Um estudo realizado em Pernambuco constatou que adolescentes ( $\leq 19$ anos) apresentavam chance 1,89 vez maior de interromper precocemente do AME, enquanto que as mulheres entre 20 e 29 anos mostraram chance 1,27 vez maior quando comparadas com aquelas $\geq 30$ anos (SILVA CS, et al., 2017).

Esse achado pode ser explicado pela possível insegurança das adolescentes quanto à habilidade de amamentar e também pelo fato de essas serem mais propensas a erros de introdução alimentar, o que pode estar associado ao baixo poder aquisitivo ou à repetição do hábito alimentar, que em muitos casos se apresenta inadequado. Enfim, mulheres com mais idade possuem maiores possibilidade de manter a AME até os seis meses por conta da maior experiência e maior conhecimento acerca da amamentação (SILVA CS, et al., 2017).

Outro fator de risco relacionado ao desmame precoce é o trauma mamilar e a dor durante a prática de amamentar (GONÇALVES GP, et al., 2019). Fissuras e rachaduras na aréola podem surgir nos primeiros dias de amamentação, tais problemas geralmente são ocasionados pelo ato da sucção ou pela pega incorreta do lactente (LIMA APC, et al., 2018). Os traumas mamilares/dor devem ser notados pela equipe de saúde como marcadores de dificuldades e podem ser evitados quando se adotam medidas profiláticas durante as consultas de pré-natal, momento em que há a oportunidade de promoção e incentivo à amamentação (ALVARENGA SC, 2017).

Destarte, é importante uma maior adesão, por parte das gestantes e lactantes, às ações de aconselhamento sobre 0 aleitamento, principalmente para as primíparas. Estas ações partem preferencialmente da Unidade Básica de Saúde (UBS), objetivando principalmente a promoção do aleitamento materno e prevenção de agravos decorrentes da interrupção desta prática (ANDRADE HS, et al., 2018).

Dentre os fatores ambientais que mais surgiram, o mais predominante foi o retorno ao trabalho que apareceu em $42 \%$ dos artigos pesquisados, dentro das variáveis ambientais foi a mais prevalente. $\mathrm{Na}$ literatura, fica claro que a introdução de novos alimentos na dieta do lactente inicia-se justamente no período do término da licença maternidade (CRISTOFARI RC, et al., 2019; LIMA APC, et al., 2018).

Isso ocorre devido à necessidade financeira familiar que fazem com que as mulheres tenham que ajudar nas despesas de casa e em alguns casos até assumir o papel de chefes de famílias (ALVARENGA SC, 2017). Outro fator muito comum, é a interferência familiar, em uma pesquisa realizada na Indonésia, foi 
constatado que mães que moram em famílias extensas (mais de cinco familiares em uma casa) têm mais chances de ocorrer o desmame precoce que nas famílias nucleares (HAS EMM, et al., 2018).

Esta relevante evidência respalda a importância do profissional se relacionar com o ambiente familiar da nutriz, conciliando sua assistência com a rede social da mesma, valorizando as ações em saúde, o vínculo, reconhecendo o papel determinante da família dentro dessa prática, trazendo-a para junto de si, para que o cuidado em saúde seja em prol do aleitamento materno, pois entendendo toda a bagagem familiar materna, permite uma maior adesão e manejo dessa prática (DIAS RB, et a., 2016).

Assim sendo, a função do enfermeiro, é de suma importância. Pois é o profissional que atua na assistência direta as mulheres e crianças, no âmbito hospitalar e comunitário. Possuindo papel importante na promoção e proteção ao aleitamento materno, por meio do fortalecimento de ações comunitárias, reorientação dos serviços de saúde, orientações as gestantes e puérperas e na formação e articulação de redes de apoio a esta prática (MONTESCHIO CAC, et al., 2015). Além de desenvolver habilidades para identificar quais crenças estão atreladas a prática de cada nutriz, assim como quais delas podem ou precisam ser mantidas, modificadas, reestruturadas ou ressignificadas.

O enfermeiro utiliza-se da promoção, para educação de adultos, favorecendo um ambiente acolhedor, onde as mulheres e familiares possam expor suas crenças, dúvidas e sentimentos. No entanto, Figueiredo MCD, et al., (2015) em sua pesquisa observou uma baixa adesão ao AME no primeiro semestre de vida, independente do fato de as genitoras terem ou não recebido orientação quanto a essa prática. Isso ocorre por dois motivos: o primeiro é à falta de incentivo dos profissionais de Saúde e o segundo é que informações entregues sem uma estratégia técnico-científica, por si só não é sinônimo de eficácia na amamentação (LIMA APC, et al., 2018; PÉREZ MRG, et al., 2016).

Quando uma orientação é realizada de forma desconexa a realidade da mãe, a mesma se torna ineficaz. $\mathrm{E}$ um dos motivos para isso se deve a um despreparo dos profissionais envolvidos (CALVACANTE AVSON, et al., 2019). Pela falta de experiência e/ou conhecimento, muitas vezes as angústias maternas são banalizadas, os fatores familiares e econômicos da mãe são desconsiderados e o vínculo de confiança entre profissional e nutriz não é formado (MARINHO MS, et al., 2015; OLIVEIRA CS, et al., 2015).

Portanto, a assistência de enfermagem deve fundamentada em teorias baseadas na atualidade, direcionando o modo do cuidar. E quanto mais capacitados estes profissionais forem, menores são as chances das mulheres se sentirem desamparadas e maiores são as de se sentirem empoderadas (OLIVEIRA CS, et al., 2015).

\section{CONSIDERAÇÕES FINAIS}

A partir da síntese dos diferentes estudos evidenciou que há uma diversidade de fatores que podem influenciar no desmame precoce e na introdução de outros alimentos antes dos seis primeiros meses de vida do lactente, correlacionando-se aos múltiplos contextos em que as mães estão inseridas. Sendo função do enfermeiro e dos demais profissionais da saúde identificar as famílias vulneráveis e mais propensas a um desmame precoce, pois a participação conjunta entre as nutrizes e seus familiares desde as consultas de pré-natal, bem como no acompanhamento da gestação e na amamentação são inquestionáveis para o sucesso da referida prática.

\section{REFERÊNCIAS}

1. ALVARENGA SC, et al. Fatores que influenciam o desmame precoce. Aquichan, 2017; 17(1): 93-103.

2. ANDRADE HS, et al. Fatores relacionados ao desmame precoce do aleitamento materno. Revista Brasileira de Medicina de Família e Comunidade, 2018; 13 (40): 1-11.

3. BARONA C, et al. ¿Mejora el bono de desarrollo humano la lactancia materna exclusiva en Ecuador? Estudios Sociales. Revista de Alimentación Contemporánea y Desarrollo Regional, 2015; 23(45): 63-81.

4. BASAIN VALDÉS JM, et al. Duración de lactancia materna exclusiva, estado nutricional y dislipidemia en pacientes pediátricos. Revista Cubana de Pediatria, 2015; 87(2): 156-166. 
5. BRASIL. Ministério da Saúde. Secretaria de Atenção à Saúde. Departamento de Atenção Básica. Cadernos de Atenção Básica. Saúde da criança: aleitamento materno e alimentação complementar. Brasília: Ministério da Saúde; 2015.

6. BRASIL. Ministério da Saúde. Secretaria de Atenção a Saúde. Departamento de Ações Programáticas e Estratégicas. Pesquisa de Prevalencia de Aleitamento Materno em municípios Brasileiros. Brasília (DF): Ministério da Saúde; 2010.63p.

7. CALVACANTE AVSON, et al. Avaliação dos conhecimentos e práticas em aleitamento materno dos profissionais de saúde em um hospital amigo da criança. Revista Brasileira de Educação e Saúde, 2019; 9(2): 13-20.

8. CAMPOS MAS, et al. Prática de aleitamento materno exclusivo informado pela mãe e oferta de líquidos aos seus filhos. Revista Latino-Americana de Enfermagem, 2015; 23(2): 283-290.

9. CRISTOFARI RC, et al. Conhecimento acerca do aleitamento materno de gestantes atendidas na atenção básica de saúde. Revista Brasileira em Promoção Saúde, 2019; 32: 1-10.

10. DIAS RB, et al. Conhecimento de enfermeiras e estratégias de incentivo da participação familiar na amamentação. Ciencia e Saude Coletiva, 2016; 21(8): 2527-2536.

11. FIGUEIREDO MCD, et al. Banco de leite humano: o apoio à amamentação e a duração do aleitamento materno exclusivo. Journal of Human Growth and Development, 2015; 25 (2): 204-210.

12. GONÇALVES GP, et al. Fatores que contribuem para a interrupção do aleitamento materno exclusivo e complementar. RESU - Revista Educação em Saúde, 2019; 7(2): 233-239.

13. HAS EMM, et al. Early weaning food for infants (0-6 months old) in madurese people based on transcultural nursing theory. IOP Conference Series: Earth and Environmental Science, 2018; 116 (1): 0-7.

14. LIMA APC, et al. A prática do aleitamento materno e os fatores que levam ao desmame precoce: uma revisão integrativa. J. Health Biol Sci, 2018; 26 (6): 189-196.

15. MACIEL VBS, et al. Aleitamento materno em crianças indígenas de dois municípios da Amazônia Ocidental Brasileira. Acta Paul Enferm, 2016; 29 (4): 469-475.

16. MARGOTTI E, MATTIELLO R. Fatores de risco para o desmame precoce. Revista da Rede de Enfermagem do Nordeste, 2016; 17(4): 537-544.

17. MARINHO MS, et al. A Atuação Do (A) Enfermeiro (A) Na Promoção, Incentivo e Apoio Ao Aleitamento Materno: Revisão da Literatura. Revista Enfermagem Contemporânea, 2015; 4(2): 189-198.

18. MONTEIRO JNS, et al. Fatores associados à interrupção precoce do aleitamento materno exclusivo em prematuros. Arquivos Catarinenses de Medicina, 2020; 49(1): 50-65.

19. MONTESCHIO CAC, et al. O enfermeiro frente ao desmame precoce na consulta de enfermagem à criança. Revista Brasileira de Enfermagem, 2015; 68(5): 869-875.

20. OLIVEIRA CS, et al. Amamentação e as intercorrências que contribuem para o desmame precoce. Revista Gaúcha de Enfermagem, 2015; 36: 16-23.

21. ORGANIZAÇÃO MUNDIAL DA SAÚDE (OMS). Infant and young child feeding: model chapter for textbooks for medical students and allied health professionals. Geneva: WHO; 2009.

22. PANG WW, et al. Direct vs. Expressed breast milk feeding: Relation to duration of breastfeeding. Nutrients, 2017; $9(6): 1-13$.

23. PÉREZ RRG, et al. Tiempo de lactancia materna exclusiva y estructura familiar: Duration of exclusive breastfeeding and the family structure. Revista Cubana de Pediatría, 2016; 88(1): 43-54.

24. PRADO CVC, et al. Desmame Precoce Na Perspectiva De Puérperas: Abordagem Dialógica. Texto Contexto Enferm, 2016; 25(2): 1-9.

25. QUISPE ILANZO MP, et al. Características y creencias maternas asociadas al abandono de la lactancia materna exclusiva. Revista Cubana de Salud Publica, 2015; 41(4): 582-592.

26. RAMIRO GONZÁLEZ MD, et al. Prevalence of breastfeeding and factors associated with the start and duration of exclusive breastfeeding in the Community of Madrid among participants in the ELOIN. Anales de Pediatría (English Edition), 2018; 89(1): 32-43.

27. SANTO FA, et al. Conhecimento de mães sobre formas de aleitamento e hábitos deletérios. Distúrb Comun, 31(4): 641-650.

28. SILVA CS, et al. Association between postpartum depression and the practice of exclusive breastfeeding in the first three months of life. Jornal de Pediatria (Versão em Português), 2017; 93(4): 356-364.

29. TAVEIRO EAN, et al. Adesão Ao Aleitamento Materno Exclusivo Em Bebês De 0 a 6 Meses Nascidos Em Um Hospital E Maternidade Do Município De São Paulo. Revista Brasileira de Ciências da Saúde, 2020; 24(1):71-82. 